\title{
Production of polyhydroxyalkanoate biopolymer from vinasse using Ralstonia eutropha
}

\author{
Eduardo dos Santos*, Claudia Gai, Kellen Zanfonato, Francielli Martinhago, Willian Steffen, Luci Kelin Quines, \\ Melodi Schmidt, Willibaldo Schmidell, Glaucia Aragão \\ From 5th Congress of the Brazilian Biotechnology Society (SBBIOTEC) \\ Florianópolis, Brazil. 10-14 November 2013
}

\section{Background}

Brazil is the world's leading ethanol producer and exporter. Due to increasing fuel demands, ethanol production has escalated in recent years. Vinasse is generated as a byproduct of the ethanol industry and is mainly used as fertilizer. The excessive usage of vinasse in soil leads to groundwater contamination [1].

The objective of this project is to develop a fermentation process with the bacterium Ralstonia eutropha for the production of polyhydroxyalkanoates (PHAs) using vinasse as the main nutrient and carbon source. This process will enable the utilization of vinasse for production of biobased, biodegradable $\mathrm{P}(3 \mathrm{HB})$ biopolymer. These biopolymers are intracellularly accumulated by $R$. eutropha as carbon and energy reserves. The potential applications of $\mathrm{P}$ $(3 \mathrm{HB})$ as alternatives to petroleum-based plastics are abundant. In this work, pure vinasse was successfully used as substrate for the production of $\mathrm{P}(3 \mathrm{HB})$ with the achieved accumulation reaching $50 \%$ of cell mass as polymer.

\section{Methods}

The microorganism used was Ralstonia eutropha (DSM545).

Vinasse was collected at Usina Iracema (Iracemapolis, São Paulo, Brazil). A 50L sample was taken directly after the distillation process, sterilized by filtration and stored at $-20^{\circ} \mathrm{C}$.

In order to analyze the $\mathrm{P}(3 \mathrm{HB})$ production, two precultures were prepared, one with NB and a second with vinasse with addition of urea [2].

Batch fermentation was done using a New Brunswick Scientific ${ }^{\mathrm{TM}}$ reactor with a working volume of $4.0 \mathrm{~L}$, with no nitrogen feed. $\mathrm{pH}$ was corrected using $2.5 \mathrm{M} \mathrm{NaOH}$ and $2.7 \mathrm{M} \mathrm{HCl}$ solutions. Culture was followed during 22 hours.

Determination of biomass, nitrogen, and protein content were done according to [2]. The composition of the vinasse was determined by HPLC, as well as the final concentration of $\mathrm{P}(3 \mathrm{HB})$, according to [3] with modifications.

\section{Results and conclusions}

Previous results from our group suggested no inhibition effect of vinasse on $R$. eutropha growth and the strain can grow with a maximum growth rate of $0,27 \mathrm{~h}^{-1}$.

The analysis of the vinasse revealed that its main components are glycerol, fructose, saccharose and mannitol. These substrates can be utilized by the bacteria. According to the experimental analysis, the microorganism barely used fructose to grow and accumulate biopolymer. Saccharose and mannitol, on the other hand, were consumed entirely. $25 \%$ of the glycerol was consumed.

The nitrogen concentration when the culture started was 0.063 g. $\mathrm{L}^{-1}$, which indicates nitrogen limitation since the beginning of the culture. As a consequence, $\mathrm{P}(3 \mathrm{HB})$ was accumulated during all culture to a concentration of around $50 \%$ during the culture. This is low compared to $80 \%$ (gPHB/gBiomass) in defined media [4] but promising from a waste product. According to the carbon balance, $79 \%$ of the calculated biomass and biopolymer production was reached from vinasse, indicating the feasibility of the process.

\section{Acknowledgements}

We thank Capes and CNPq for the financial aid to this project through the AJT fellowship awarded to C.S.Gai. We also thank our collaborators Prof A.J. Sinskey and his team from MIT. 


\section{References}

1. Laime EMO, Fernandes DCS, Freire EA: Possibilidades tecnológicas para a destinação da vinhaça: uma revisão. Revista Trópica - Ciências Agrárias e Biológicas 2011, 5(16).

2. Aragão GMF, Lindley ND, Uribelarrea JL, Pareilleux A: Maintaining a controlled residual growth capacity increases the production of polyhydroxyalkanoate copolymers by Alcaligenes eutrophus. Biotechnol Lett 1996, 18:937-942.

3. Karr DB, Waters JKA, Emerich DW: Analysis of poly- $\beta$-hydroxybutyrate in Rhizobium japonicum bacteroids by ion-exclusion high- pressure liquid chromatography and UV detection. Appl Environ Microbiol 1983, 46:1339-1344.

4. Reinecke F, Steinbüchel A: Ralstonia eutropha strain $\mathrm{H} 16$ as model organism for PHA metabolism and for biotechnological production of technically interesting biopolymers. J Mol Microbiol Biotechnol 2009, 16:91-108.

doi:10.1186/1753-6561-8-S4-P132

Cite this article as: dos Santos et al:: Production of

polyhydroxyalkanoate biopolymer from vinasse using Ralstonia eutropha. BMC Proceedings 2014 8(Suppl 4):P132.

\section{Submit your next manuscript to BioMed Central} and take full advantage of:

- Convenient online submission

- Thorough peer review

- No space constraints or color figure charges

- Immediate publication on acceptance

- Inclusion in PubMed, CAS, Scopus and Google Scholar

- Research which is freely available for redistribution

Submit your manuscript at www.biomedcentral.com/submit 